\title{
Postoperative Delirium Following Orthognathic Surgery in a Young Patient
}

\author{
Fernanda Herrera da Costa, DMD, ${ }^{*}$ Paulo Adilson Herrera, MD, MSc, $\dagger$ \\ Cecília Luiz Pereira-Stabile, DMD, MSc, PhD, $\ddagger$ and \\ Glaykon Alex Vitti Stabile, DMD, MSc, PhD $\oint$
}

\begin{abstract}
Delirium is an organic mental syndrome with acute onset characterized by diffuse brain dysfunction and neural activity disorganization. It is usually related to cognition and perception changes, decreased level of consciousness, and disorganization of thoughts that are unrelated to previous dementia. Occurrence is more frequent in patients with previous degenerative disease and elderly patients, especially those older than 85 years. Although the pathophysiology is not totally known, studies have shown that, among the main factors that lead to delirium, the drugs used for general anesthesia induction are the most relevant (hypnotics, anticholinergic drugs, and $\mathrm{H}_{2}$ receptor blockers), especially those used in long surgical procedures. This report describes the case of a 24-year-old woman with a noncontributory medical and psychological history. She underwent bimaxillary orthognathic surgery with a total general anesthesia time of 7 hours. Postoperatively, she developed agitation, confusion, and delirium. After a psychiatry consult and discussion with the anesthesia team, the diagnosis of psychotic break owing to late postoperative delirium was established.

(C) 2016 Published by Elsevier Inc on behalf of the American Association of Oral and Maxillofacial Surgeons

J Oral Maxillofac Surg 75:284.e1-284.e4, 2017
\end{abstract}

Orthognathic surgery is a complex and specialized procedure that might require long periods of general anesthesia. Postoperative delirium (POD) is a common complication involving postoperative disturbance of the mental state, especially in patients older than 85 years, with an incidence of 15 to $53 \%$ in the elderly population. ${ }^{1}$ However, the number of occurrences increases as the procedure lengthens, such as heart and orthopedic procedures, ${ }^{2}$ with an even higher incidence when associated with pre-existing mental disorders. ${ }^{3}$ School-age children appear to be at risk, with an incidence of $12 \%$ when undergoing eye surgery. Its occurrence in adolescents and young adults is rare. ${ }^{4}$ In addition to age, risk factors possibly associated with POD are Parkinson disease, malnutrition, head trauma, prolonged anesthesia, infection, and metabolic disorders. ${ }^{1,5}$

POD is an acute-onset mental syndrome characterized by global cognitive dysfunction: a lower level of consciousness, abnormal attention, increase or decrease in psychomotor activity, and dysfunction of the sleep-and-wake cycle. ${ }^{6}$ The etiology is multifactorial, and the most accepted theory is that occurrence is related to changes in neurotransmission caused by drugs, neurologic conditions, underlying diseases, environmental factors, trauma, or longer exposure to general anesthesia., ${ }^{2,7}$ The drugs most commonly involved in the development of delirium are

\footnotetext{
*Surgeon, Department of Oral and Maxillofacial; MSc Student, Department of Oral Pathology, State University of São Paulo, São Paulo, SP, Brazil.

$\dagger$ Private Anesthesiologist, Hospital Evangélico de Londrina, Londrina, PR, Brazil.

$\ddagger$ Associate Professor, Department of Oral Medicine and Pediatric Dentistry, State University of Londrina, Londrina, PR, Brazil.

$\S$ Adjunct Professor, Department of Oral Medicine and Pediatric Dentistry; Coordinator, Residency in Oral and Maxillofacial Surgery, State University of Londrina, Londrina, PR, Brazil.
} 
narcotics, hypnotics, $\mathrm{H}_{2}$ receptor blockers, drugs for the treatment of Parkinson disease, anticholinergic agents, and corticosteroids. The potential for drugs to induce delirium depends on underlying risk factors and individual susceptibility. ${ }^{1,8}$ Thus far, the occurrence of POD in association with orthognathic surgery has not been reported in the literature.

Through the report of a clinical case, the aim of this article was to describe and characterize POD as a possible complication of orthognathic surgery.

\section{Report of Case}

This study was approved by the institutional review board of the Hospital Evangélico de Londrina (Londrina, Brazil) and the patient signed an informed consent agreement.

A 24-year-old woman was referred by her orthodontist to the oral and maxillofacial surgery team for an orthognathic evaluation. She denied having any health issues except for a diagnosis of von Willebrand disease. The patient was professionally active in the preoperative period and did not present with any mental disorders or systemic comorbidities. Two hours before surgery, as recommended by her hematologist, she received a transfusion of coagulation factor VIII. As a preanesthetic drug, midazolam $15 \mathrm{mg}$ was taken orally. Upon arrival in the operating room, cefazolin $1 \mathrm{~g}$ and dexamethasone $10 \mathrm{mg}$ were administered intravenously. Anesthesia was induced by intravenous injection of propofol, remifentanil, and rocuronium, maintained by isoflurane inhalation and continuous infusion of remifentanil, and monitored by a mass spectrometer. During the procedure, the patient was continuously monitored by electrocardiography, radial artery puncture, capnography, and a bispectral index (BIS) monitor. Mean arterial pressure was maintained at 65 to $80 \mathrm{mmHg}$ with the aid of a $\beta$-blocker (metoprolol) and the BIS was kept at 40 to 60 . During the procedure, the patient's electroencephalogram did not show any suppression periods. There was no need for blood transfusion during surgery or the postoperative period.

After anesthetic induction, the patient underwent orthognathic surgery for correction of a Class II dentofacial deformity through maxillary impaction, mandibular advancement, and an advancement genioplasty. Surgery was uneventful. The patient remained under general anesthesia for 7 hours.

After extubation and postanesthetic recovery, the patient was transferred to the floor, where she was prescribed an intravenous antibiotic (cephalothin), a corticosteroid (hydrocortisone), and 2 nonsteroidal anti-inflammatory and analgesic medications (ketorolac and dipyrone).

During the first 24 hours after surgery, the patient was calm, oriented in time and space, communicative, and responsive to verbal commands. She did not exhibit postoperative hemorrhage, was able to take liquids orally, had spontaneous diuresis, moderate swelling, and adequate pain control, and was discharged home the day after surgery. Upon discharge, she was prescribed an oral antibiotic and nonsteroidal analgesic. No further steroids were prescribed.

Within the first 48 hours, the patient's parents contacted the surgical team reporting unusual behavior. She alternated periods of agitation and aggressiveness and was unable to sleep. As time passed, the symptoms became more severe and she became incoherent, presenting delusions of control and thought insertion. She also reported an inability to breathe during sleep and constant thoughts about dying. At the same time, her parents observed an increase in aggressiveness. As a result, the patient was not sleeping adequately and eating precariously.

She was referred for psychiatric emergency services. After rigorous clinical evaluation concurrently with the anesthesiology team, the patient was diagnosed with a psychotic break caused by POD, described in the International Classification of Diseases, Tenth Revision as a psychotic disorder from the use of multiple drugs and other psychoactive substances (code F19.5). ${ }^{9}$ Treatment consisted of:

- Instructing the caregivers not to leave the patient alone while the symptoms were present

- Prescription of antidepressant drugs (escitalopram and mirtazapine) and an anxiolytic (diazepam), with instructions to increase the dose in case of worsening symptoms

Within the first 24 hours after starting the prescribed medication, the patient showed considerable improvement of mood and signs, and symptoms of paranoid delirium became less severe.

After 3 days of using psychiatric medication, the patient was completely asymptomatic and the drugs were gradually discontinued. At 10 months after surgery, she was totally asymptomatic and resumed all her professional and social activities uneventfully.

\section{Discussion}

The purpose of this report was to characterize POD as a possible complication of orthognathic surgery. According to Lipowski, ${ }^{6}$ POD can be classified by its time of occurrence as emergence delirium, when it occurs within the first 24 hours after anesthesia, and late delirium, when it occurs after a lucid interval of at least 1 day. ${ }^{10}$ Other investigators have classified POD, based on its clinical presentation, as agitation or hyperactivity, characterized by an increased state of alertness to external stimuli, or silent or 
hypoactivity, characterized by a decreased response to stimuli and introversion. ${ }^{10}$ The latter classification is more relevant for instructional purposes, because the patient can present an overlap of signs and symptoms, with alternating episodes of agitation and lethargy. 6,10

The present case had a diagnosed episode of a psychotic break originating as late POD, because the first symptoms were reported after hospital discharge. The patient had episodes of agitation and hyperactivity, with an altered sleep-and-wake cycle.

The pathophysiology of POD is not fully understood, but the most accepted theory holds that delirium is a neuropsychiatric manifestation caused by disturbances in brain metabolism and neurotransmitters. ${ }^{6,10,11}$ Acetylcholine appears to be the major neurotransmitter that leads to delirium, ${ }^{12,13}$ because its cholinergic action is related to the regulation of attention, memory, and sleep. ${ }^{10,11}$ Other neurotransmitters might be involved in the state of delirium, such as dopamine, serotonin, glutamate, and $\gamma$-aminobutyric acid. ${ }^{13}$ Other accepted theories state that the presence of certain cytokines (interleukin-1, interleukin-6, and tumor necrosis factor- $\alpha$ ) during infection and ultimately levels of cortisol in patients under constant stress, anxiety, and trauma increase serotonin levels and can cause delirium ${ }^{2,9}$ (Table 1). In addition, there are reports linking the continuous use of corticosteroids with delirium. ${ }^{8,14}$

An orthognathic procedure leads to changes in brain homeostasis, because of the drugs used in general anesthesia. It also can lead to an increase in cortisol levels as an effect of constant stress and anxiety related to surgery. These combined effects can eventually alter the correct functioning of involved neurotransmitters, causing POD. In the present case, the patient received only 3 doses of hydrocortisone, all within 24 hours. Therefore, corticosteroids as a probable cause of delirium are unlikely, because studies have suggested that psychiatric symptoms during corticosteroid therapy are dose dependent. ${ }^{15,16}$

\section{Table 1. MAIN HYPOTHESES FOR THE DEVELOPMENT} OF DELIRIUM

Factors Associated With Delirium

Increase in level of cortisol from stress

Increase in dopamine transmission

Increase in noradrenergic transmission

Decrease in cholinergic transmission

Increase in serotonergic transmission

Increased transmission of $\gamma$-aminobutyric acid receptors Increased inflammation from cytokines

da Costa et al. Delirium After Orthognathic Surgery. J Oral Maxillofac Surg 2017.

The diagnosis of delirium is clinical and must follow the guidelines established by the American Psychiatric Association as described in the Diagnostic and Statistical Manual for Mental Disorders (Table 2). In addition, secondary symptoms can be considered additional diagnostic tools, such as agitation or psychomotor retardation, disorientation, attention or perception disorders, and changes in the sleep-andwake cycle, as measured by the Confusion Assessment Method. The final diagnosis is confirmed by a psychiatrist to establish the etiology of the condition.

The prevention of POD consists mainly of preoperative risk assessment. It is extremely important to conduct a thorough review of the physical and mental status of the patient. However, because the pathophysiologic mechanisms of delirium are not fully understood, there are no reliable preoperative interventions to prevent its occurrence. Strategies aim to control factors that might trigger the condition, in addition to intensive monitoring during surgery of the BIS, with prompt correction of hypoxia, uncontrolled hypotension, and electrolyte imbalances. ${ }^{17}$ The present case is extremely unusual because the

\section{Table 2. DIAGNOSTIC CRITERIA FOR DELIRIUM ACCORDING TO THE AMERICAN PSYCHIATRIC ASSOCIATION'S DSM AND THE CAM}

$\begin{array}{lc}\begin{array}{l}D S M \\ \text { for Diagnostic Criteria }\end{array} & \text { CAM Diagnostic Criteria } \\ \text { for Delirium }\end{array}$

Disturbance of consciousness with difficulty to focus, maintain, or switch attention

Cognitive disorders or perception changes

Acute onset with fluctuating symptoms throughout the day

Evidence that the disorder was caused by a medical condition, medications, drugs, abstinence, or other such conditions

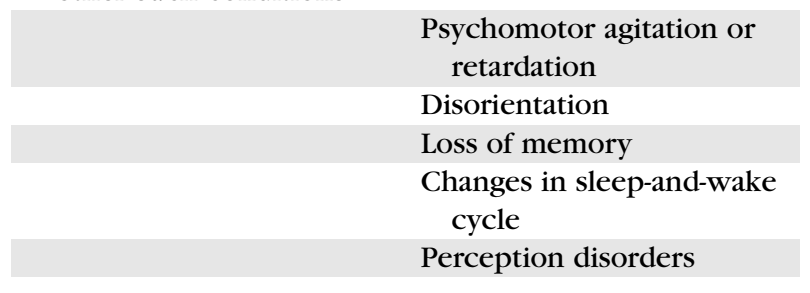

Abbreviations: CAM, confusion assessment method; DSM, diagnostic and statistical manual for mental disorders.

da Costa et al. Delirium After Orthognathic Surgery. J Oral Maxillofac Surg 2017.

Acute and fluctuating onset of symptoms

Disturbance of attention

Disorganized thoughts

Altered consciousness

Psychomotor agitation or retardation

Changes in sleep-and-wake cycle

erception disorders 
patient was young, had no personal or family history of mental disorders, did not take any medications before surgery, and underwent general anesthesia uneventfully under strict monitoring.

Adequate evaluation by a psychiatrist is necessary for the diagnosis and treatment of POD. Cognitive and pharmacologic interventions are usually indicated for acute symptom control. ${ }^{18,19}$ In the present patient, antipsychotic drugs and nonpharmacologic methods were used.

POD is a common complication in geriatric patients undergoing longer surgeries. However, there are no reported cases involving younger patients after orthognathic surgery. Therefore, it has not previously been described as a possible postoperative anesthetic complication. POD is a potentially severe complication of long surgeries with a multidisciplinary diagnosis and treatment involving the maxillofacial surgeon, anesthesiologist, and psychiatrist.

\section{References}

1. Ruiz-Neto P, Moreira N, Furlaneto M: Postanesthetic delirium Rev Bras Anestesiol 52:242, 2002

2. Inouye SK: Delirium in older persons. N Engl J Med 354:1157, 2006

3. Reubne B, Yoshikawa T, Besdine R: Geriatrics review syllabusA core curriculum in geriatrics medicine. Am Geriatr Soc 3a:120, 1996

4. Voepel-Levis T, Malviya S, Toit AR: A prospective cohort study of emergence agitation in the pediatric postanesthetic care unit. Anesth Analg 96:1625, 2003
5. Inouye S, Viscoli C, Hormitz R: A predictive model for delirium in hospitalized elderly medical patients based on admission characteristics. Ann Intern Med 119:474, 1993

6. Lipowski ZJ: Delirium (acute confusional state). JAMA 258:1789, 1987

7. Han L, McCusker J, Cole M, et al: Use of medications with anticholinergic effect predicts clinical severity of delirium symptoms in order medical inpatients. Arch Intern Med 8:1099, 2001

8. Rome HP, Braceland FJ: The psychological response to ACTH, cortisone, hydrocortisone, and related steroids substances. Am J Psychiatry 108:641, 1952

9. Caetano D (ed): Classificação de Transtornos Mentais e de Comportamento da CID-10: Descrições Clínicas e Diretrizes Diagnósticas. Porto Alegre, Brazil, Artes Médicas, 1993

10. O'Keefe ST, Chonchubhair A: Postoperative Delirium in the elderly. Br J Anaesth 73:673, 1994

11. Lindsay J, Macdonald A, Starke I: Delirium in the Elderly. Oxford, UK, Oxford University, 1990

12. Chan D, Brennan N: Delirium: Making the diagnosis, improving the prognosis. Geriatrics 54:28, 1999

13. Mach J, Dysken M, Kuskowiski M: Serum anticholinergic activity in hospitalized elderly with delirium. A preliminary study. J Am Geriatr Soc 43:491, 1995

14. Inouye S, van Dyck C, Alessi C, et al: Clarifying confusion. The Confusion Assessment Method: A new method for detection of delirium. Ann Intern Med 113:941, 1990

15. Warrington TP, Bostwick JM: Psychiatric adverse effects of corticosteroids. Mayo Clin Proc 81:1361, 2006

16. Brown ES, Chandler PA: Mood and cognitive changes during systemic corticosteroids therapy. Prim Care Companion J Clin Psychiatry 3:17, 2001

17. Rozner M: Preoperative prediction of postoperative delirium. JAMA 20:1573, 1994

18. Richeimer S: Psychological intervention in delirium: An important component of management. Postgrad Med 81: 173,1987

19. Jacobson S, Schreibman B: Behavioral and pharmacological treatment of delirium. Am Fam Physician 56:1997, 2001 